Dieses Manuskript ist

zu zitieren als

Angew. Chem. Suppl.

$1983,501-526$
This manuscript is

to be cited as

Angew. Chem. Suppl.

$1983,501-526$

O Verlag Chemie GmbH, D-6940 Weinheim, 1983

$0721-4227 / 83 / 0505-0501802.50 / 0$

Addition eines Phospha-alkins an Metall-Metall-Mehrfachbindungen ${ }^{* *}$

Von Gerd Becker, Wolfgang A. Mermann", Wallibald Kalcher, Gangolf W.

Kriechboum, Claudid Pahl, C. Thoous Wamer und Manfred L. Ziegler

Nach der Entdeckunq des unsubstituierten Phosphha-acetylens (Methylidinphosphans, $\mathrm{H}-\mathrm{C} \equiv \mathrm{P}$ ) durch $\mathrm{G}:$ er $/ 1 \mathrm{a} /$ und der Phospharet?incyanine durch Dimroth und Hof foann / $/ \mathrm{b} /$ hat die Cherie des niedervalenten Phosphors mit der erfolgreschen Syn-

- Prot. Dr. W.A. Herrmann ${ }^{[+]}$, W. Kalcher, G.w. Kriechbaur

Institut $f 0 r$ Anorganische Chemie der Johann Wolfgang coethe-Universitat, Niederurseler Hang, D-6000 Frankfurt an Main 50

Prot. Dr. M.L. Ziegler, C. Pahl

Anorganisch-chemisches Institut der Universitát Heidelberg, Im Neuenheimer Feld, D-6900 Heudelberg :

Prof. Dr. G. Becker, C.T. Wagner

Institut for Anorganische Chemse der Universitst Stuttgart Pfaffenwaldring 55, D-7000 stuttgart

$[+]$ Korrespondenzautor

* xoeplexchesile reaktiver organischer Verbindungen, 42. Diese Arbeit wurde van der Deutschen Forschungsgebeinschaft und den Ponds der Chesuschen Industrie grobzogig unterstotzt. 41. Matteil.: W.A. Bermesn, M.L. Ziegler. O.Serhadl2, Organoeetallics, in Druck.

\title{
$-501-$
}

these teils uberraschend bestandiger Phospha-alkene (A) und Phospha-alkine (B) neue Dimensionen in der Chemie der Hauptgruppenelemente erschlossen /2/. Neuerdings beginnt sich auch<smiles>CP=C1CC1</smiles>

A
$\mathrm{IP} \equiv \mathrm{C}-$

B

eine reichhaltige Komplexchemie dieser Substanzklassen abzuzeichnen $/ 3,4 /$. Systematische Untersuchungen uber möglıche metallinduzierte Reaktionen von $\mathrm{p}-\mathrm{C}$-Mehrfachbindungen sind jedoch nicht erkennbar. So sind bisher weder Voraussagen uber den Koordinationsmodus konstitutionell intakter Phospha-alkenbzw. Phospha-alkin-Liganden Fcolich noch hat man über Folgereaktionen komplexierter $\mathrm{p}-\mathrm{C}$-Liaanden berichtet. Ist mit Acetylenen und Nitrilen die metallzentrierte Fetathese unter Spaltung der jewelligen Dreifachbindungen prinzipiell moglich 15/, so hat man die P-C-Dreifachbindung in solche Untersuchungen ebensowenig exnbezogen wie die P-C-Doppelbindung als moglichen Analogiefall der olefan-Metathese.

Im Kontext unserer Arbeiten uber die Chemie von Metall-Metall-Mehrfachbindungen haben wir ansbesondere im Hinblick auf mögliche Cycloadditions- und/oder Metathese-Reaktionen das nach Becker, Gresser und uhl bequem zugăngliche 2.2-Dimethylpropylidinphosphan (1)/6/ exemplarisch auf sein Verhalten gegenuber Metall-Metall-Mehrfachbindungen gepruft. 
Das Phospha-alkin I addiert sich Im Tomperaturbereich $-60, \ldots+25 \%$ rasch und quantitativ sowohl an die Rhodium-Rhodiun-Doppelbindung als auch an die Molybdan-Molybdan-Dreifachbindung der Zweikernkomplexe $2 \mathrm{a}$ bzw. 2b (C1. 1, 2), In beiden fallen werden kristalline, luft- und feuchtigkeitsbeständige produkte jg bzw. ํㅐ

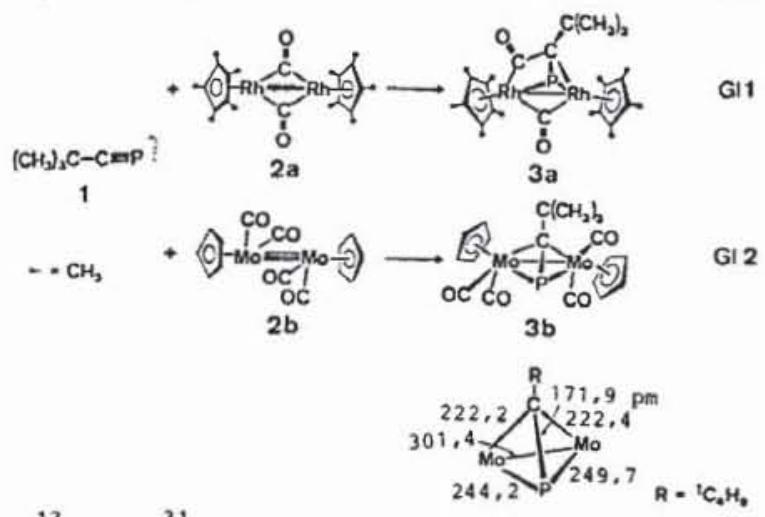

${ }^{1} \mathrm{H}-,{ }^{13} \mathrm{C}$ - und ${ }^{31} \mathrm{P}-$ Kernresonanzspektroskopie gesichert ist

(Tab. 6). Die zusammensetzung der neuen Komplexverbindungen

folgt aus den Elementaranalysen sowle den FD-Massenspektren

(vgl. Experimenteller Teil). Hinsichtlich der Bildungsweise und des Konstitutionstyps gleichen die Additionsprodukte $3 \mathrm{a}$ und $3 \mathrm{~b}$ den entsprechenden, auf analoge Wesse zuganglichen Alkin-Derivaten /7-9/. Die metalianduzierte Spaltung der Phosphor-Kohlenstoff-Bindung wird nicht beobachtet. Die thermische Eliminierung der intakten Phospha-alkin-Bausteine aus den Komplexderivaten $3 \mathrm{a}$ und $3 \mathrm{~b}$ ist einer GC-Analyse zufolge nicht möglich.

Mit der Adation des Phospha-alkins an die Rhodiun-RhodiumDoppelbindung von $2 a$ geht eine Insertion dieses Liganden in eine der beiden Metall-Carbony1-Funktionen einher. Dabe1 bildet sich regiospezifisch das cyclische strukturelement der Verbindung 3a. Stbchiometrisch und stereochemisch vö112g ana$\log$ war die von uns beschrlebene Umsetzung von Phenylacetylen mit der reaktıven Rhodıum-Verbındung 2a verlaufen /8/. Das metallorganische Phospha-alkin-Derivat $3 \mathrm{a}$ besitzt nach Ausweis der ${ }^{13} \mathrm{C}$-NMR-Spektren eine starre Molekulgeometrie: Die beiden chemsch nuchtäquivalenten Carbonylgruppen weisen bex tiefen Temperaturen ebenso wie bei Rauntemperatur getrennte Signalsátze auf (Abb. 2). Bel den analoaen Alkın-Komplexen liegen vereinzelt fluktuierende strukturen vor $17,8 /$.

Der Molybdan-Komplex $3 b$ we1st die rontgenstrukturanalytisch ermittelte Geometrie eines metallorganischen Tetrahedrans auf (Abb. 1, Tab. 1-5). Der an die Mehrfachbindung addierte cpBaustein liegt senkrecht uber der Molybdan-Molybdän-Bindung; letztere werst aufgrund des zu 301.4(0) pm gefundenen Metal1abstandes den Charakter einer Einfachbındung auf (Formelschema); Vergleichsdaten hierzu finden sich in Lit. $/ 10-13 /$. Das P(1)$c(5)-c(6)-s k e l e t t$ ist nicht wie im freien phospa-alkin 1inear /16/, sondern infolge seiner Komplexierung Uber die Dresfachbindung gewinkelt $\left(3 P(1), C(5), C(6)=127.9(3)^{\circ}\right.$; Tab. 4). Erwartungsgemá ist die $c(5)-C(6)-B$ intung von der Komplexierung nicht betroffen $(154.5(6) \mathrm{pm})$.

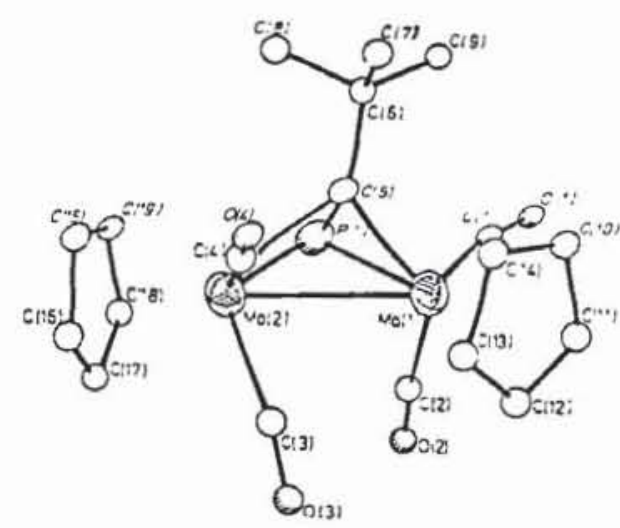

Abb. 1. Struktur des $u, n^{2}$-Phosphaalkin-komplexes 3 b. Die Wasserstof $f-$ atcose sind der Obersichtilichkeit halber weggelassen.

Das Herzstuck des Molekuls ist exn Dimetallatetrahedran-Gerust, welches annahernd $c_{s}$-symmetrie besitzt; die spiegelebene ist durch die Ligandatome $P(1), C(5)$ und $C(6)$ definiert. Wie aus dem Pormelschema hervorgeht, weisen die bexden Metallatome zun C- bzw. P-Atom jewes1s identische Abstando auf (vg1. Tab. 4). Die verbleibenden Liganden $\left(\mathrm{CO}, \mathrm{C}_{5} \mathrm{H}_{5}\right)$ ordnen sich nach sterischen Erfordernissen so an, daB inre gegenseltigen AbstoBungen möglichst gerıng werden (Abb. 1). Samtliche CarbonylGruppen folgen den Anforderungen des strenq termanalen Koordinationstypus; alle Molybdän-CO- und $\mathrm{C}-\mathrm{O}-\mathrm{B}$ indungslangen stimmen miteinander innerhalb der Signifikanzgrenze (30) uberexn. Keine strukturchemischen Besonderheiten weisen erwartungs-

\section{- 505 -}

gemar $B$ die zentrisch gebundenen Cyclopentadienyl-Lıganden auf. die miteinander einen Winkel von $39.2^{\circ}$ einschlieben (Tab. 5).

Das Phosphoratom $P(1)$ 1st pyramidal konfiguriert und weist eine Winkelsumme von $197.3^{\circ}$ auf. Die Basizităt dieses Atons konnte unlangst durch Sekundarkomplexserung mit dem Organometallfragment $w(C O)_{5}$ demonstriert werden $/ 4 c ;$. Die Bindungswinkel des Phosphoratoms $P(1)$ sinc nicht viel groBer als jene 1n. der Struktur des weiBen Phosphors $\left(\mathrm{P}_{4} ; 60^{\circ} / 14 /\right)$.

we ein Vergleich mit strukturanalogen, ebenfalls dem Tetrahedran-Typ zugehorıgen Molekulen zeigt (Tab. 7), hat die Inkorporierung des Phospha-alkıns 1 in die Organometall-Verbindung $2 \underline{b}$ nicht nur mit dem erzielten Strukturtyp $\underline{c}$, sondern auch mit dem AusmaB der dabel erfolgten Geometrieverănderungen der Komponenten glexche Konsequenzen wie die entsprechenden Alkin-Additionen: Die Phosphor-Kohlenstoff-Bindung ( $\mathrm{P} \equiv \mathrm{C}-\mathrm{Ab}-$ stand in 1: $153.6(2) \mathrm{mm} / 16 /)$ erfahrt nicht die formal zu erwartende Aufweitung bis zur E1nfachbindung (182-187 pm; $/ 2,15,16 /)$.

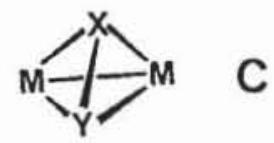

sondern west mit einer Lange von $171.9(3)$ pm den Charakter einer typischen Doppelbindung auf: die zugehbrigen Kovalenzradien betragen $100 \mathrm{pm}$ (Phosphor) und $67 \mathrm{pm}$ (Kohlenstoff) /15/. Die $\mathrm{P}-\mathrm{C}$-Abstande in authentischen Phospha-alkenen wurden bisher zu 168 bis 172 pm gefunden /2/. Auch bes der Addition von 
Alkinen $(C \equiv C)$, Carbin-Komplexen $(M \geq C)$ und analogen Liganden (z.B. As $\equiv A s)$ an die Metall-Metall-Bindungen von 2h und vergleichbaren zweikernkomplexen wird ubereinstimmend nur eine Doppelbindung erzielt (Tab. $7 ; 113,17-22 /$ ).

Die vorliegende Arbeit weist erstmals auf die enge komplexchemische Analogie zwischen Alkinen und Phospha-alkinen hin, inden sie uber die glatte, unter B1ldung eines neuartigen :letallacyclus sowle elnes metallorganischen Tetrahedran-Holekuls verlaufende Addition von Neopentylidinphosphan (1) an reaktive Metal1-Metall-Mehrfachbindungen berichtet. Die Ausdehnung dieses of fenbar auch in der Chenle der Phosphor-KohlenstoffVerbindungen erfolgrolchen Syntheseprinzips auf phospha-alkene und silaolefine bietet sich an und ist Gegenstand kunftiger Untersuchungen.

\section{Experimente11er Te11}

Alle Arbeiten wurden unter Luft- und Feucht1gkeitsausschluB durchgefuhrt (Schlenk-Technik; absolutierte, $\mathrm{N}_{2}$-gesattigte LSsungsmittel; trockene $\mathrm{N}_{2}$-Atmospharre). Bezüglich der allgemelnen Arbeltstechnik sel auf fruhere Experimentalarbeiten dieser Reihe $/ 23,24 /$ sowie die Vorbemerkungen der Prăparatevorschriften in Lit. /25/ verwiesen. Schmelz- und zersetzungspunkte wurden in abgeschmolzenen kapillaren ermittelt und sind nicht korriglert (BUch1 SMP-20). Als stationare Phase bel der săulenchromatograph1schen Aufarbeitung der Komplexe $3 a \underline{i}$, $\mathrm{N}_{2}$-gesatt1gtes Florisil (Merck 12999; 100-200 mesh) verwendet.

Neopentylidinphosphan (1) wurde nach Becker et al. dargestellt 16/; das Prăparat enthielt noch ca. 20 \& Hexamethyldistloxan, welches aber weder mat $2 \mathrm{a}$ noch mat 2 p p reagiert und in Hochvakuum oder durch săulenchromatographle aus den Rohprodukten abgetrennt werden kann.

\section{Synthese des Cycloaddukts za:}

Man kUhlt eine Losung von $226 \mathrm{mg}(0.5 \mathrm{mmol})$ 2a in $30 \mathrm{ml}$ Tetrahydrofuran in einem Aceton/Trockeneis-Bad auf $-60^{\circ} \mathrm{C}$ ab und versetzt diese dann mit ubersehussigem 2.2-Dimethylpropylidinphosphan $(1) ; c a .0 .1 \mathrm{~m} 1)$, das mit Hexamethyldisiloxan verunreinigt sein darf. Dabei schlagt die Losungsfarbe in Sekundenfrist von violett nach rotbraun um. Zur Vervollstăndigung der Reaktion 1aBt man noch 15 min ruhren, entfernt das Kuh1bad und zieht schlie81ich die fluchtigen Antelle im olpumpenvakuum be1 $+25^{\circ} \mathrm{C}$ ab. Der braune Ruckstand wird in möglichst wenig Toluol gelost und uber eine mit Florisil/Toluol beschickte Chromatographiesalule $(1=20 \mathrm{~cm} ; \emptyset=1.2 \mathrm{~cm})$ bel $-20{ }^{\circ} \mathrm{C}$ filtriert; die Komplexverbindung $3 \mathrm{a}$ wird dabei mit einem aus Toluol und Diethylether (50/50 Vol. -8$)$ bestehenden Laufmittelgemisch eluiert. Nach dem Einengen des rotbraunen Eluats und Kristallisation des Rllekstands aus n-Hexan/Diethylether $\left(1: 1 ;-40^{\circ} \mathrm{C}\right)$ erhrlt man das analysenreine Produkt 3a. Ausbeute $270 \mathrm{mg}(85 \mathrm{8})$. Rotbraune, Luftstab1le Kristalle, die sich ab $180^{\circ} \mathrm{C}$ unter Schwarzfarbung allmahlich zersetzen und bei $186-188^{\circ} \mathrm{C}$ schmelzen. 3a 1st in n-Hexan und Petrolether
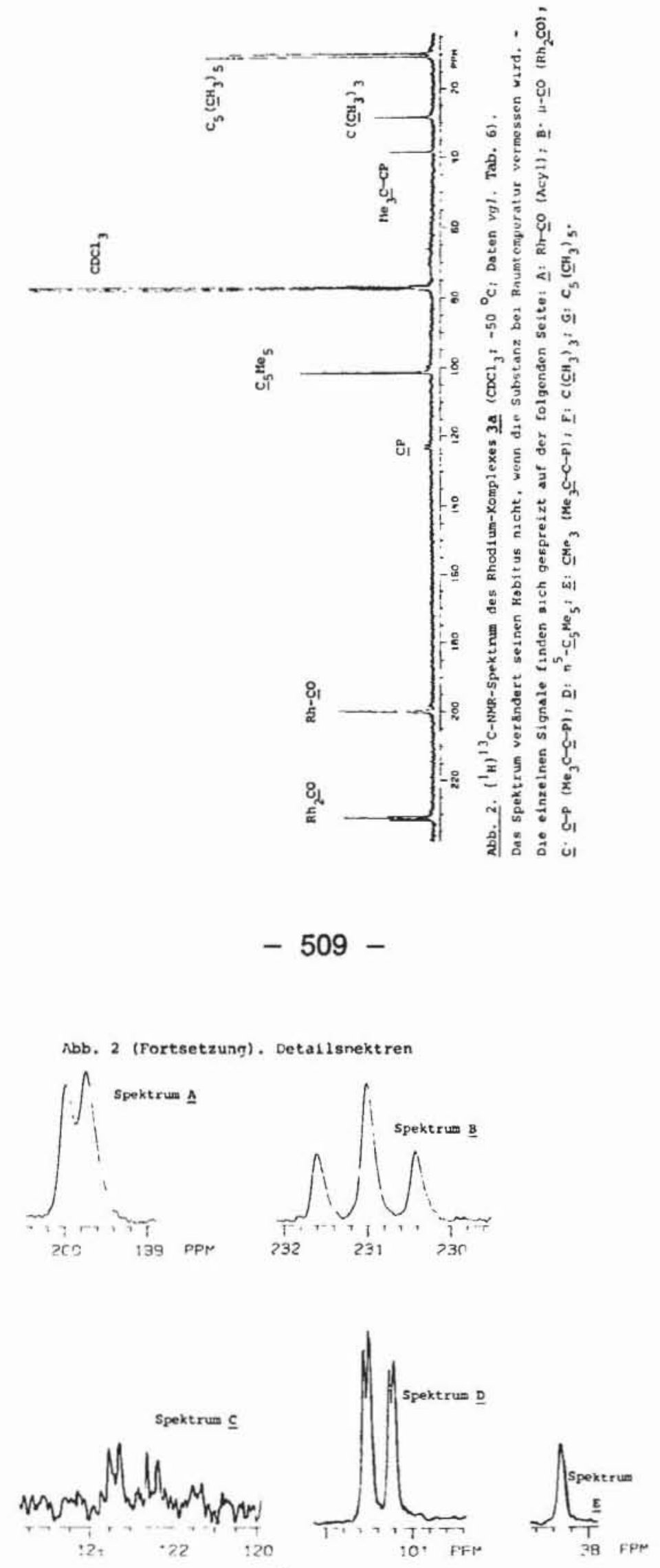
maBig, in Toluol gut und in stärker polaren organischen Solventien (z.B. Methylenchlorid, Tetrahydrofuran) sehr gut 10s11ch. Die Losungen sind an Luft nur kurzzeitıg haltbar.

$\mathrm{C}_{27} 7_{39} \mathrm{O}_{2} \mathrm{PRh}_{2} \quad(632.39)$

Ber. C 51.28 \& 6.22 P 4.90

cef. C 51.58 H 6.39 P 5.00

Molmasse 632 (FD-Massenspektrum; Tolvol-Losung)

2. $\left(\mu, n^{2}\right.$-Neopenty 11 dinphosphan) bis Idicarbony $1\left(n^{5}\right.$-cyclopentad1eny 1) molybdăn (Mo- - Mo) $3 b$ :

Eine Lösung von $434 \mathrm{mg}(1.0 \mathrm{mmol})$ des Molybdăn-Komplexes $2 \mathfrak{p}$ in $50 \mathrm{ml}$ Tetrahydrofuran wird bel $-78^{\circ} \mathrm{C}$ (Trockeneis/AcetonKUhlbad) m1t Ubersch(ssigem 2.2-Dimethylpropyl1dinphosphan (1) versetzt (ca. $0.4 \mathrm{ml})$. Unter magnetischem Ruhren $1 \mathrm{abt}$ mar: die tiefrote Lðsung sich langsam auf Raumtemperatur erwärmen und entfernt nach 2 h das Solvens im Wasserstrahlvakuum. Den 811gen, roten Ruckstand trocknet man zur Beseitigung von uberschüssigem $\underset{2}{2}$ sowie von Hexamethyldisiloxan im Hochvakuum, um thn schlleblich als n-Hexan-Suspension einer mit Florisil/nHexan beschiclten wassergekthlten Chromatographiesăule aufzutragen $(1=60 \mathrm{~cm} ; \emptyset=1.8 \mathrm{~cm})$. Der Phospha-alkin-Komplex $3 \underline{\underline{b}}$ wird mit Toluol/n-Hexan $(1: 2)$ als rote zone elusert. Mit Tetrahydrofuran als Laufmittel erhalt man anschlieBend noch geringe Mengen (< $10 \mathrm{mg}$ ) einer grunen, carbonylhaltigen Verbindung, deren zusammensetzung bisher nicht geklart werden konnte. Der feste, rote Rllkstand des Toluol/n-Hexan-Eluats

\section{$-511-$}

liefert bei der Unkristallisation aus Diethylether/n-Hexan $\left(1: 4 ;-40^{\circ} \mathrm{C}\right)$ analysenreines $3 \mathrm{~b}$. Ausb. $430 \mathrm{mg}(81 \mathrm{~s})$. Die Verbindung ist in kristaliiner Form luftbestlindig, ihre Lösungen zersetzen sich jedoch an der Luft rasch. Zers. $247^{\circ} \mathrm{C}$ unter heft1ger Gasentwacklung.

$\mathrm{C}_{19} \mathrm{H}_{19} \mathrm{MOO}_{2} \mathrm{O}_{4}^{\mathrm{P}} \quad(534.06)$

Ber. C 42.73 म 3.56 Mo 35.93 P 5.80

Gef. C 42.63 н 3.46 Mo 35.70 P.5.96

Molmasse 526 (FD-Massenspektrum; Toluol-Lôsung; Bezugsisotop ${ }^{92}$ Mo)

\section{Röntgenst rukturanalyse}

Ein Kristall dex Verbindung $3 \mathfrak{E}$ wurde in der üblichen weise auf einem Gonıometerkopf befestigt; er war kleiner $(0.5 \times 0.2 \times 0.3 \mathrm{mal})$ als die optimale Kristallaicke ( $t$ opt $=\frac{2}{\mu}=0.15 \mathrm{~cm}$ ). Die Bestinung der Gitterkonstanten erfolgte nach der Methode der kleinsten Quadrate /29/ aus diffraktobetrisch bestimaten $Q$-Werten von 60 ausyewahlien Reflexen. Die Intensitaten worden ebenfalls mit dew AED-S1emens ( $\theta-2 \theta$-Abtastung, 5-Wert-Methode) gemessen; dabei wurden lediglich Reflexe mit I>2.50(I) berücksichtıgt. Diese wurden LP-korrigiert; esne Absorptionskorrektur murde nicht durchgef thrt.

Die Lagepararter der Mo-Atome wurden einer 3d-Pattersonsynthese, die der rest1_chen Atume (außer H) 3d-Fourier-bzw. Differenzfouriersynthesen entnomen. Die Wasserstoffatone wurden wit dea Programm H-FIX /26/ erhalten. Dabei wurden die Methylgruppen als starre Gruppen $(d C-\mathbb{B}=96 \mathrm{pa})$ angesehen und alle Wasserstoffatobe isotrop mit einea gemeinsamen U-Wert (U) $=\mathrm{B} / 8 \mathrm{x}^{2}$ ) verfeinert. Die $\mathrm{H}$-Atowe der cyclopentadienylringe vurden in einea zyklus individuell ait isotropen U-werten verfesnert, wobes letztere das 1,2-fache der V-Werte der korrespondierenden kohlenstoffatcoe waren.

Die Strukturverfeinerung erfolgte nach der Methode der kleinsten guadrate. Alle Rechnungen basieren auf dem Progrannsystem SHEIXTL /26/, die Atomformfaktoren mirden den International Tables for $x$-Ray Crystallography /27/ entncesen. Die isotrope Verfeinerung ergab $R=0.060$, die anisotrope Eit cewichtung der strukturasplituden $R_{v}=0.027$ $\left(R_{v}=\Sigma\left|P_{o b s} F_{c}\right| \sqrt{\text { wht }} / 2 F_{\text {obs }} \sqrt{\text { wht }}\right.$, wght $\left.=1 / \sigma^{2}(F)\right)$. Der "Coodness-of-fit"Wert $\left(\left(\left[\Sigma_{w}\left(\left|p_{\text {obs }}\right|-\left|r_{c}\right|\right)^{2}\right\} /(n-p)\right\}^{\frac{2}{2}}\right)$ betragt $2.548(n-3484, p=245)$. Die Atoakoordinaten aind in Tab. 2, Temperaturfaktoren in Tab. 3, Abstande und winkel in tab. 4 enthalten. Daten zu einigen besten Ebenen sind in Tab. 5 aufgefohrt. Die Atcobezeichnungen beziehen sich auf Nbb. 1 .

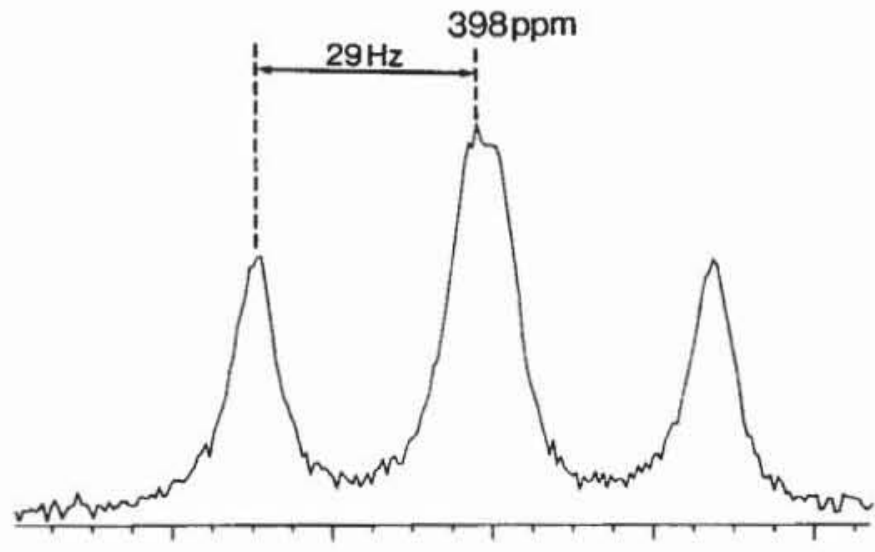

Nhb.3. ${ }^{31}$ P-NerR-Spektrum des Rhodium-Komplexes 3 A (Daten vgl. Tab. 6).

Tab. 1. Kristallographische Daten des $\mu, n^{2}$-Phosphaacetylen-Komplexes 3p

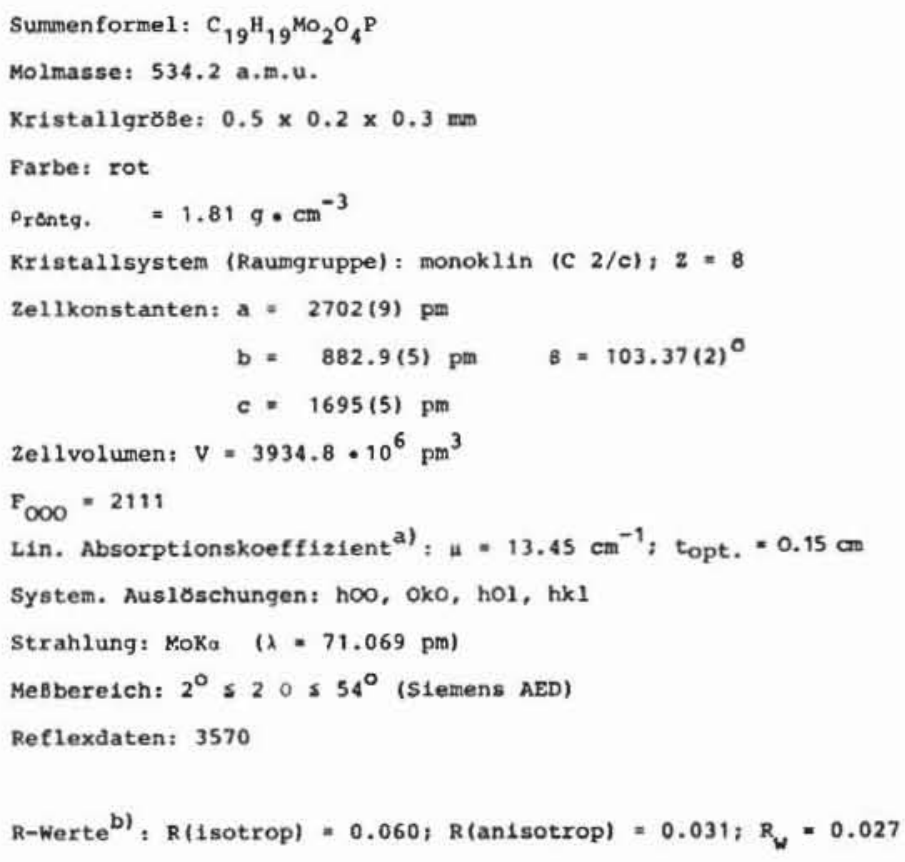

a) Eine Absorptionskorrektur wurde nicht vorgenommen.

b) $R_{w}=\frac{\sum\left|F_{c}-F_{c b s}\right| \sqrt{\text { wght }}}{\sum F_{\text {cbs }} \sqrt{\text { wght }}} ;$ wght $=\frac{1}{\sigma^{2}(F)}$ 
Tab. 2. Atomkoordinaten (1n Bruchtellen der Zel1konstanten, $\left.\times 10^{4}\right)$ und isotrope Temperaturfaktoren $\left(\times 10^{2}\right)$ des $4, n^{2}$-phos phaacetylen-Koroplexes $3 \mathrm{~b}$

\begin{tabular}{|c|c|c|c|c|}
\hline Atom & $x / a$ & $y / b$ & $z / c$ & $\begin{array}{l}U_{\text {equiv. }} \\
\left.\left(U_{B}\right)^{a}\right)\end{array}$ \\
\hline $\operatorname{Mo}(1)$ & $1626(1)$ & $323(1)$ & $790(1)$ & $35(1)$ \\
\hline Mo (2) & $1094(1)$ & $-2241(1)$ & $-241(1)$ & $35(1)$ \\
\hline$P(1)$ & $693(1)$ & $-97(1)$ & $276(1)$ & $43(1)$ \\
\hline $0(11)$ & $1116(1)$ & $2932(4)$ & $1561(2)$ & $93(2)$ \\
\hline$O(2)$ & $1382(1)$ & $2450(4)$ & $-708(2)$ & $85(1)$ \\
\hline$o(3)$ & $1903(1)$ & $-901(4)$ & $-1092(2)$ & $64(1)$ \\
\hline $0(4)$ & $1906(1)$ & $-4366(4)$ & $813(2)$ & $70(11)$ \\
\hline$c(1)$ & $1286(2)$ & $1931(5)$ & $1281(3)$ & $59(2)$ \\
\hline$c(2)$ & $1459(2)$ & $1633(5)$ & $-164(2)$ & $52(1)$ \\
\hline$c(3)$ & $1622(1)$ & $-1313(4)$ & $-719(2)$ & $45(1)$ \\
\hline$C(4)$ & $1611(1)$ & $-3521(4)$ & $449(2)$ & $47(1)$ \\
\hline$c(5)$ & $1043(1)$ & $-1347(4)$ & $963(2)$ & $38(1)$ \\
\hline$c(6)$ & $908(2)$ & $-2018(5)$ & $1729(2)$ & $52(2)$ \\
\hline$c(7)$ & $130^{9}(2)$ & $-3069(6)$ & $2202(3)$ & $93(3)$ \\
\hline$c(8)$ & $416(2)$ & $-2914(8)$ & $1473(3)$ & $121(3)$ \\
\hline$c(9)$ & $830(2)$ & $-742(5)$ & $2290(3)$ & $70(2)$ \\
\hline$c(10)$ & $2254(2)$ & $507(6)$ & $1978(3)$ & $70(2)$ \\
\hline$c(11)$ & $2363(2)$ & $1549(5)$ & $1395(3)$ & $65(2)$ \\
\hline$c(12)$ & $2477(1)$ & $696(6)$ & $773(3)$ & $63(2)$ \\
\hline$c(13)$ & $2440(1)$ & $-827(6)$ & $949(3)$ & $64(2)$ \\
\hline$C(14)$ & $2303(2)$ & $-944(6)$ & $1679(3)$ & $72(2)$ \\
\hline$c(15)$ & $613(2)$ & $-4391(5)$ & $-593(3)$ & $71(2)$ \\
\hline$c(16)$ & $902(2)$ & $-4167(5)$ & $-1176(3)$ & $69(2)$ \\
\hline$c(17)$ & $718(2)$ & $-2851(6)$ & $-1600(3)$ & $74(2)$ \\
\hline$c(18)$ & $333(2)$ & $-2266(6)$ & $-1281(3)$ & $80(2)$ \\
\hline$c(19)$ & $271(2\}$ & $-3236(7)$ & -65313 & $76(2)$ \\
\hline$H(7 A)$ & 1362 & -3936 & 1890 & $97(6)$ \\
\hline$H(7 B)$ & 1210 & -3397 & 2684 & $97(6)$ \\
\hline $\mathrm{H}(7 \mathrm{C})$ & 1618 & -2494 & 2349 & $97(6)$ \\
\hline
\end{tabular}

Fortsetzung Tab. 2 .

\begin{tabular}{lrrrl} 
Atom & $x / a$ & $Y / b$ & $z / c$ & $U_{H}$ \\
\hline$H(8 A)$ & 426 & -3723 & 1099 & $97(6)$ \\
$H(8 B)$ & 162 & -2190 & 1225 & $97(6)$ \\
$H(8 C)$ & 333 & -3315 & 1953 & $97(6)$ \\
$H(9 A)$ & 569 & -93 & 1986 & $97(6)$ \\
$H(9 B)$ & 1141 & -183 & 2453 & $97(6)$ \\
$H(9 C)$ & 728 & -1107 & 2762 & $97(6)$ \\
$H(10)$ & 2163 & 759 & 2477 & 85 \\
$H(11)$ & 2359 & 2634 & 1428 & 77 \\
$H(12)$ & 2566 & 1095 & 298 & 76 \\
$H(13)$ & 2500 & -1658 & 618 & 78 \\
$H(14)$ & 2250 & -1874 & 1941 & 86 \\
$H(15)$ & 649 & -5218 & -216 & 86 \\
$H(16)$ & 1173 & -4799 & -1264 & 82 \\
$H(17)$ & 839 & -2422 & -2041 & 91 \\
$H(18)$ & 142 & -1363 & -1457 & 97 \\
$H(19)$ & 30 & -3101 & -322 & 92
\end{tabular}

a) ${ }_{\text {uequiv. }}=\frac{1}{3} \tilde{\mathrm{v}}, \mathrm{mit} \tilde{\mathrm{U}}=$ Spur der diagonalisierten U-Natrix; die $\mu$-Atane wurden mat den Program $4-F I X^{26)}$ festgelogt; sie wuxden isotrop mit einem goneinsamen $\mathrm{U}_{\mathrm{H}}$-wert $\left.\mathrm{U}_{\mathrm{H}}=\mathrm{B} / \mathrm{B}^{2}\right)$ verfeinert.

Tab. 3. An1sotrope Temperaturfaktoren $\left(\times 10^{3}\right)$ des $4, \eta^{2}=$ Phospha-alkin-K omplexes $3 \mathrm{~b}^{\mathrm{a})}$

\begin{tabular}{|c|c|c|c|c|c|c|}
\hline Atom & $v_{11}$ & $v_{22}$ & $\mathrm{v}_{33}$ & $\mathrm{v}_{23}$ & $v_{13}$ & $v_{12}$ \\
\hline Mo (1) & $36(1)$ & $32(1)$ & $35(1)$ & $0(1)$ & $7(1)$ & $-3(1)$ \\
\hline $\operatorname{Mo}(2)$ & $39(1)$ & $31(1)$ & $34(1)$ & $-3(1)$ & $10(1)$ & $1(1)$ \\
\hline$P(1)$ & $35(1)$ & $42(1)$ & $51(1)$ & $-7(1)$ & $9(1)$ & $5(1)$ \\
\hline$o(1)$ & $122(3)$ & $56(2)$ & $118(3)$ & $-34(2)$ & $61(2)$ & $1(2)$ \\
\hline$O(2)$ & $114(3)$ & $63(2)$ & $73(2)$ & $31(2)$ & $13(2)$ & $5(2)$ \\
\hline $0(3)$ & $65(2)$ & $72(2)$ & $64(2)$ & $9(2)$ & $39(2)$ & $-2(2)$ \\
\hline$O(4)$ & $65(2)$ & $62(2)$ & $84(2)$ & $19(2)$ & $18(2)$ & $24(2)$ \\
\hline$c(1)$ & $74(3)$ & $42(2)$ & $65(3)$ & $-8(2)$ & $25(2)$ & $-8(2)$ \\
\hline$c(2)$ & $60(3)$ & $42(2)$ & $53(2)$ & $4(2)$ & $12(2)$ & $O(2)$ \\
\hline$c(3)$ & $48(2)$ & $41(2)$ & $46(2)$ & $-2(2)$ & $11(2)$ & $6(2)$ \\
\hline$c(4)$ & $51(2)$ & $44(2)$ & $50(2)$ & $2(2)$ & $19(2)$ & $3(2)$ \\
\hline$c(5)$ & $42(2)$ & $35(2)$ & $39(2)$ & $-7(2)$ & $13(2)$ & $-6(2)$ \\
\hline$c(6)$ & $63(3)$ & $55(2)$ & $46(2)$ & $-4(2)$ & $28(2)$ & $-16(2)$ \\
\hline$c(7)$ & $152(6)$ & $74(4)$ & $71(3)$ & $37(3)$ & $62(4)$ & $26(4)$ \\
\hline$c(8)$ & $139(6)$ & $172(7)$ & $66(3)$ & $-13(4)$ & $50(4)$ & $-102(5)$ \\
\hline$c(9)$ & $97(4)$ & $73(3)$ & $50(3)$ & $-5(2)$ & $38(3)$ & $7(3)$ \\
\hline$c(10)$ & $61(3)$ & $99(4)$ & $44(2)$ & $-6(3)$ & $-1(2)$ & $-19(3)$ \\
\hline$c(11)$ & $52(3)$ & $60(3)$ & $75(3)$ & $-12(3)$ & $-3(2)$ & $-18(2)$ \\
\hline$c(12)$ & $37(2)$ & $77(3)$ & $73(3)$ & $11(3)$ & $11(2)$ & $8(2)$ \\
\hline$c(13)$ & $36(2)$ & $65(3)$ & $85(4)$ & $3(3)$ & $0(2)$ & $6(2)$ \\
\hline$c(14)$ & $53(3)$ & $72(3)$ & $76(3)$ & $25(3)$ & $-16(2)$ & $-7(2)$ \\
\hline$c(15)$ & $88(4)$ & $52(3)$ & $68(3)$ & $-8(2)$ & $7(3)$ & $-32(3)$ \\
\hline$c(16)$ & $85(3)$ & $54(3)$ & $67(3)$ & $-29(2)$ & $18(3)$ & $-1(3)$ \\
\hline$c(17)$ & $101(4)$ & $71(3)$ & $41(2)$ & $-5(2)$ & $2(3)$ & $-25(3)$ \\
\hline$c(18)$ & $74(3)$ & $65(3)$ & $79(4)$ & $-25(3)$ & $-30(3)$ & $8(3)$ \\
\hline$c(19)$ & $57(3)$ & $94(4)$ & $75(3)$ & $-33(3)$ & $11(3)$ & $-21(3)$ \\
\hline
\end{tabular}

a) D1e anisotropen Temperaturfaktoren sind definiert als $T=\exp \left(4\left(U_{21} b^{2} a^{* 2}+U_{22} k^{2} b^{* 2}+U_{1}{ }^{2} l c^{* 2}+2 U_{23} k b^{*} c^{*}+\right.\right.$ $\left.\left.2 \mathrm{U}_{1}, \mathrm{hla}^{*} \mathrm{C} *+2 \mathrm{U}_{12} \mathrm{~h}^{*} \mathrm{a}^{*} \mathrm{~b}^{*}\right)\right\}$

$$
-517-
$$

Tab. 4. Ausgewahlte Strukturparameter der Verbsnđung 3b

\section{A) Bindungsiangen $[\mathrm{pm}]$}

\begin{tabular}{|c|c|c|c|}
\hline $\operatorname{Mo}(1)-\operatorname{Mo}(2)$ & $301.4(0)$ & $c(5)-c(6)$ & $154.5(6)$ \\
\hline $\operatorname{Mo}(1)-P(1)$ & $249.7(1)$ & $c(6)-c(7)$ & $150.8(6)$ \\
\hline Mo $(2)-P(1)$ & $244.2(1)$ & $c(6)-c(8)$ & $152.2(7)$ \\
\hline Mo $(1)-C(5)$ & $222.4(4)$ & $c(6)-c(9)$ & $152.0(6)$ \\
\hline $\operatorname{Mo}(2)-C(5)$ & $222.2(4)$ & & \\
\hline \multirow[t]{2}{*}{$P(1)-C(5)$} & $171.9(3)$ & $\operatorname{Mo}(1)-C(10)$ & $231.8(4)$ \\
\hline & & $\operatorname{Mo}(1)-C(11)$ & $228.9(4)$ \\
\hline $\operatorname{Mo}(1)-C(1)$ & $197.5(4)$ & $\operatorname{Mo}(1)-C(12)$ & $232.9(4)$ \\
\hline $\operatorname{Mo}(1)-C(2)$ & $195.5(4)$ & $\operatorname{Mo}(1)-C(13)$ & $238.1(4)$ \\
\hline $\operatorname{Mo}(2)-C(3)$ & $197.5(4)$ & $\operatorname{Mo}(1)-C(14)$ & $236.5(4)$ \\
\hline \multirow[t]{2}{*}{$\operatorname{Mo}(2)-C(4)$} & $195.9(4)$ & & \\
\hline & & $\operatorname{Mo}(2)-c(15)$ & $229.9(5)$ \\
\hline$C(1)-O(1)$ & $114.9(6)$ & $\operatorname{Mo}(2)-c(16)$ & $230.0(5)$ \\
\hline$c(2) \rightarrow O(2)$ & $115.1(5)$ & $\operatorname{Mo}(2)-c(17)$ & $235.5(4)$ \\
\hline$c(3)-0(3)$ & $115.3(5)$ & $\operatorname{Mo}(2)-C(18)$ & $237.8(5)$ \\
\hline$c(4)-0(4)$ & $116.0(5)$ & $\operatorname{Mo}(2)-C(19)$ & $234.1(5)$ \\
\hline$c(10)-c(11)$ & $142.9(7)$ & $c(15)-c(16)$ & $140.7(8)$ \\
\hline$c(11)-c(12)$ & $138.7(7)$ & $c(16)-c(17)$ & $139.7(7)$ \\
\hline$c(12)-c(13)$ & $138.5(7)$ & $c(17)-c(18)$ & $137.8(8)$ \\
\hline$c(13)-c(14)$ & $137.5(8)$ & $c(18)-c(19)$ & $140.6(8)$ \\
\hline$c(14)-c(10)$ & $139.5(8)$ & $c(19)-c(15)$ & $136.5(7)$ \\
\hline
\end{tabular}

B) winkel [grad)

Mo (1), P(1), Mo (2) $\operatorname{Mo}(1), C(5), M o(2)$ Mo(1), $P(1), C(5)$ Mo $(2), P(1), C(5)$ Mo (1), $C(5), P(1)$
$75.2(0) \quad M o(1), c(1), O(1)$ $85.3(1)$ Mo(1), $\mathrm{C}(2), \mathrm{O}(2)$ $60.4(1) \quad \operatorname{Mo}(2), C(3), O(3)$ $61.7(1) \operatorname{Mo}(2), c(4), O(4)$ $77.4(1) \quad P(1), M O(1), C(1)$
$175.2(4)$ $176.4(4)$ $170.2(3)$ $174.7(3)$ 74.0(1) 
Fortsetzung Tab. 4 .

\begin{tabular}{|c|c|c|c|}
\hline $\operatorname{Mo}(2), C(5), P(1)$ & $75.4(1)$ & $P(1), \operatorname{Mo}(1), C(2)$ & $77.2(1)$ \\
\hline $\operatorname{Mo}(1), \operatorname{Mo}(2), P(3)$ & $53.2(0)$ & $P(1), M o(2), C(3)$ & $104.4(1)$ \\
\hline $\operatorname{Mo}(1), \operatorname{Mo}(2), C(5)$ & $47.4(1)$ & $P(1), \operatorname{Mo}(2), C(4)$ & $123.1(1)$ \\
\hline $\operatorname{Mo}(2), \operatorname{Mo}(1), P(1)$ & $51.6(0)$ & $c(1), \operatorname{Mo}(1), c(5)$ & $90.5(2)$ \\
\hline $\operatorname{Mo}(2), \operatorname{Mo}(1), C(5)$ & $47.3(1)$ & $C(3), \operatorname{Mo}(2), C(4)$ & $90.1(2)$ \\
\hline$P(1), c(5), c(6)$ & $127.9(3)$ & $c(4), \operatorname{Mo}(2), C(5)$ & $81.0(1)$ \\
\hline $\operatorname{Mo}(1), c(5), c(6)$ & $132.5(2)$ & $c(5), c(6), c(8)$ & $108.7(3)$ \\
\hline $\operatorname{Mo}(2), C(5), C(6)$ & $135.4(2)$ & $c(5), c(6), c(9)$ & $109.6(3)$ \\
\hline$P(1), \operatorname{Mo}(2), C(5)$ & $42.9(1)$ & $c(8), C(6), C(9)$ & $109.0(4)$ \\
\hline \multirow[t]{3}{*}{$P(1), \operatorname{Mo}(1), C(5)$} & $42.2(1)$ & $C(5), C(6), C(7)$ & $113.7(4)$ \\
\hline & & $c(7), c(6), c(8)$ & $107.7(4)$ \\
\hline & & $c(7), c(6), c(9)$ & $108.0(3)$ \\
\hline
\end{tabular}

Die C,C,C-winkel der zentrisch gebundenen CyclopentadienylLiganden 11egen in Bereich 106.7-109.0 .

Tab. 6. Spektroskopische Daten der neuen Phospha-alk1n-Komplexe $3 a$ und $3 p$

\section{RHODIUM-KOMPLEX 3a}

IR $\left(v \mathrm{vCO} ; \mathrm{cm}^{-1}\right)=1965 \mathrm{~s}, 1800 \mathrm{sst}\left(\mathrm{Rh}_{2} \mathrm{CO}\right), 1755 \mathrm{sch}, 1657 \mathrm{st}-\mathrm{sst}$ (Acyl-CO), $1646 \mathrm{Sch}$ (KBr)

1805 sst, $1680 \mathrm{~m}-\mathrm{st}, \mathrm{br}$ (n-Hexan)

1792 sst, $1657 \mathrm{sch}, 1640$ st, br $\left(\mathrm{CH}_{2} \mathrm{Cl}_{2}\right)$

1 $\mathrm{H}$-NMR $\left(270 \mathrm{MHz} ; \mathrm{CDCl}_{3} ; 25^{\circ} \mathrm{C} ; \mathrm{CHCl}_{3}\right.$-Standard): $6 \mathrm{C}_{5} \mathrm{Me}_{5} 1.84$ $[\mathrm{s}, 15 \mathrm{H}], 1.92[\mathrm{~s}, 15 \mathrm{H}]: 6 \mathrm{CMe}_{3} 0.83$ [s, 9H]

$31_{\mathrm{P}-\mathrm{NHR}}\left(\mathrm{C}_{6} \mathrm{D}_{6} ; 28^{\circ} \mathrm{C} ; 1\right.$ als ext. Standard, $\left.8=0 \mathrm{ppm}\right): 6 \mathrm{n} 398 \mathrm{pm}$ $\left(t,{ }^{\prime} J(R h, P)=29 \mathrm{~Hz} ; \mathrm{Abb} .3\right)$.

$\left(1^{1}\right)^{13} \mathrm{C}-\mathrm{NMR}\left(75.4 \mathrm{MHz} ; \mathrm{CDCl}_{3} ;-50{ }^{\circ} \mathrm{C}\right.$; Probe $050,{ }^{13} \mathrm{CO}$ angereichert): $6 \mathrm{Rh}_{2} \mathrm{CO} 231.01\left[\mathrm{t},{ }^{\mathrm{J}} \mathrm{J}(\mathrm{Ph}, \mathrm{C})=44 \mathrm{~Hz}\right]$; $6 \mathrm{RhCO} 199.84$ Id, ${ }^{1} \mathrm{~J}(\mathrm{Rh}, \mathrm{C})=18 \mathrm{~Hz}$ ); $8 \underline{\mathrm{CP}} 122.8\left(\mathrm{dd},{ }^{1} \mathrm{~J}(\mathrm{Rh}, \mathrm{C}) \geqslant 18 \mathrm{~Hz},{ }^{1} \mathrm{~J}(\mathrm{C}\right.$, $\left.{ }^{13} \mathrm{c}\right) 2070 \mathrm{~Hz} 1 ; \delta \underline{C}_{5} \mathrm{Me}_{5} 101.51\left[\mathrm{~d},{ }^{1} \mathrm{~J}(\mathrm{Rh}, \mathrm{C})=4 \mathrm{~Hz}\right], 101.21[\mathrm{~d}$, $\left.{ }^{1} \mathrm{~J}(\mathrm{Rh}, \mathrm{C})=4 \mathrm{~Hz}\right]$; $\delta \underline{C}^{t}$ But $38.30[\mathrm{~s}) ; 8 \mathrm{C}\left(\mathrm{CH}_{3}\right)_{3} 28.26[\mathrm{~s}]$; ${ }^{8 C_{5}}\left(\underline{C H}_{3}\right)_{5} 10.56[\mathrm{~s}], 9.61$ [s] (Abb. 2).

MOLYBDAN-KOMPLEX $3 \mathrm{~b}$

IR (vCO; $\left.\mathrm{cm}^{-1}\right)$ : 1995 sst, 1963 sst, 1941 sst, 1921 sst,

$$
\begin{aligned}
& 1912 \text { st, } 1864 \text { sst (n-Hexan) } \\
& 1987 \text { sst, } 1959 \text { sst, } 1925 \text { sst, } 1850 \text { sst }\left(\mathrm{CH}_{2} \mathrm{Cl}_{2}\right) \\
& 1980 \text { sst, } 1918 \text { sst, br, } 1848 \text { sst (KPr) }
\end{aligned}
$$

\section{$-521-$}

Tab. 5. Geraden, beste Ebenen und Schnittwinkel des $\mu, \eta^{2}-$ Phospha-acetylen-Komplexes 쪼

\section{A) Geraden und beste Ebenen}

Gerade a: Mo(1)-Mo(2) Gerade b: Mo (1)-P(1) Gerade c: Mo (2)-P(1) Gerade d: $P(1)-C(5)$ Gerade e: $c(5)-c(6)$
Ebene A: Mo (1)-Mo(2)-P(1) Ebene B: Mo (1)-Mo $(2)-C(5)$ Ebene C: $P(1)-C(5)-C(6)$ Ebene D: $C(5)-C(6)-C(7)-C(8)-C(9)$ Ebene E: $C(10)-C(11)-C(12)-C(13)-C(14)$

\begin{tabular}{|c|c|c|c|c|c|c|c|c|c|}
\hline b & c & d & e & A & e & C & 0 & E & $F$ \\
\hline \multirow[t]{10}{*}{51.6} & 126.8 & 91.5 & 91.4 & 90.0 & 90.0 & 178.4 & 56.3 & 1204 & $162 . \theta$ \\
\hline & 75.3 & 119.5 & 79.7 & 90.0 & 130.7 & $12 a .0$ & 167.5 & $1 \in 7.7$ & 142.6 \\
\hline & & 110.1 & n.c & 90.0 & 131.8 & $5 \times, 9$ & 136.5 & $10 n .6$ & 60.5 \\
\hline & & & 51.8 & 37.4 & 19.0 & 9.0 & Sa. 5 & 40.5 & 72.8 \\
\hline & & & & 14.5 & 70.6 & 90.0 & $110 . \mathrm{C}$ & $89 . \overline{2}$ & 64.2 \\
\hline & & & & & 58.3 & 51.0 & 95.8 & 70.2 & d1.6 \\
\hline & & & & & & 91.2 & 39.5 & 38.8 & 72.9 \\
\hline & & & & & & & 83.9 & 56,4 & 10.3 \\
\hline & & & & & & & & 33.7 & 69.9 \\
\hline & & & & & & & & & 39.2 \\
\hline
\end{tabular}
Ebene F: $c(15)-C(16)-c(17)-c(18)-C(19)$ B) Schnittwinke1 [grad) a)

a) Winkel zwischen den Normalen der Ebenen.
Fortsetzung Tab. 6

1 $\mathrm{H}-\mathrm{NMR}\left(270 \mathrm{MHz} ; \mathrm{CDCl}_{3} ; 25^{\circ} \mathrm{C} ; \mathrm{CHCl}_{3}\right.$-Standard $) ; 8 \mathrm{C}_{5} \mathrm{H}_{5} 5.33$ [s, $\left.10 \mathrm{H}], 8 \mathrm{CMe}_{3} 1.20 \mathrm{~s}, 9 \mathrm{H}\right]$

$31_{\mathrm{p}-\mathrm{NMR}}\left(121.46 \mathrm{MHz}, \mathrm{C}_{6} \mathrm{D}_{6} ; 25^{\circ} \mathrm{C} ; \mathrm{Me}_{3} \mathrm{C}-\mathrm{C} \equiv \mathrm{P}\right.$ als int. Standard, $8=0 \mathrm{pps}): 8 \mathrm{P}-42.5[\mathrm{~s}, \mathrm{br}]$

$\left({ }^{1} \mathrm{H}\right)^{13} \mathrm{C}-\mathrm{NMR}$ (67.9 MHz; $\mathrm{C}_{6} \mathrm{D}_{6} ; 25^{\circ} \mathrm{C} ;$ int. TMS $)={ }^{8} \mathrm{C}_{5} \mathrm{H}_{5}$ $89.67 ; \mathrm{SCH}_{3} 38.80$ und $38.87 ; 8 \mathrm{Me}_{3} \mathrm{C} 42.99 ; 6 \mathrm{CO} 232.01,231.96$, $231.45,231.37$ 


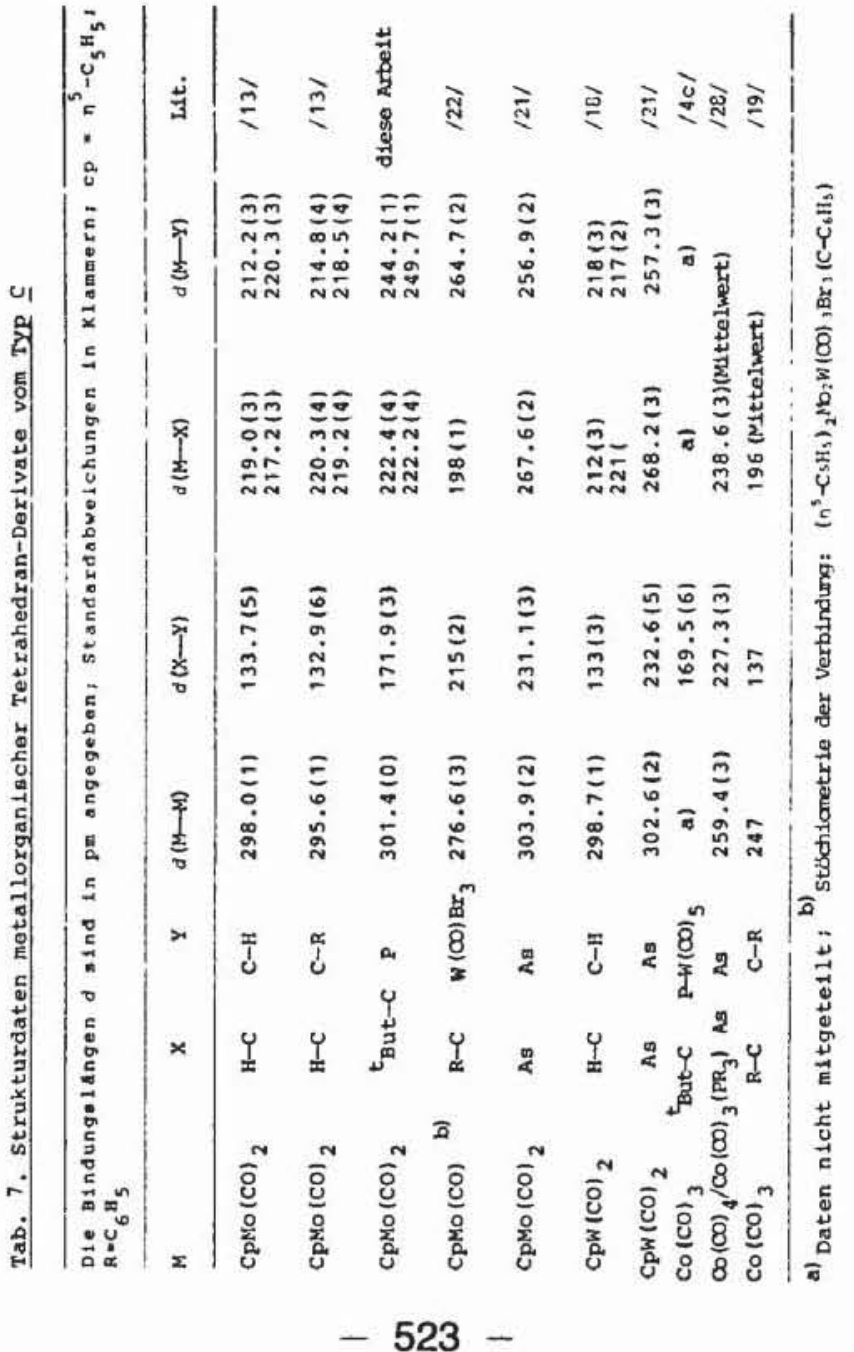

Literatur

'1/ a) T.E.Gier, J. Aner. Chem. Soc, 83 (1961) 1769; b) K.Dyrroth, P.

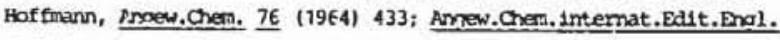
$\underline{3}$ (1964) 384; Chen. Ber. 99 (1966) 1325.

12) Zusammenfassende Darstellungen: a) R. Appel, F. Knoll, I. Ruppert, Angew.Chem. 93 (1981) 771; Angew.Chem.internat.Ed1t. Engl. 20 (1981) 731; b) G, Becker, w. Becker. 0.2undt, phosphorus and sulfur (1983), in Eruck; c) E.Fluck, Top. Thoschorus Chen. 10 (1980) 193; d) K.Dinroth, Top. Ourr. Chen. 38 (1973) 1.

13/ Phospha-alken-Komplexe: a) Th.C. Xlebach, R. Lourens, F. Blckelhaupt, C.H. Stam, A. van Herk, J.organometal. Chem. $210(1981) 211$; b) H. Eshtiagh-Hossein1, K.W. Kroto, J.F. Nixon, M.J. Maah, M.J. Taylor, J.Chem.Soc.Commun. 1981, 199; c) Th.C. van der Knaap, F. Bickelhaupt, H. van der Poel, G. van Koten, C.H. Stam, J.Amer. Chem.Soc. 104 (1982) 1756; d) H.W. Kroto, J.F. N1xon, М.H. Taylor, A.A. Frew, K.W. Muir, Polyhedron 1 (1982) 89.

14) Phospha-a $1 k_{1} n-K_{0}$ plexe: a) D. Seyferth, R.S. Henderson, J.Organometa1.Chem. 162 (1978) C 35; b) J.C.T.R. BurckettSt. Laurent, P.W. Hitcheock, H.W. Kroto, J.F. Nixon, J.Chem.Soc.Chem.Commun. 1981, 1141; c) J.C.T.R. BurckettSt. Laurent, P.B. Hitchcock, H.w. Kroto, M.F. Meidine, J.F. N1xon, J.Organometa1. Chem. 238 (1982) c 82 .

15/ R.R. Schrock, M.L. Listemann, L.G. Sturgeoff, J.Amer.Chem. Soc. $104(1982) 4291$.

16/ G. Becker, G. Gresser, W.Uh1, 2. Naturforsch. 36b (1981) 16.

17/ R.S. Dickson, G.S. Evans, G.D.Fallon, J.Organometal. Chem. 236 (1982) c 49.

18/ W.A. Herrmann, Ch. Bauer, J. Welchmann, J.organometal. Chem., im Druck.
19/ Ubersicht: W.A. Herrmann, J.Organometal.Chem. 250 (1983), im Druck.

/10/ W.A. Herrmann, G.W. Kriechbaum, M.L. 21egler, H. Pfisterer, Angew.Chem. 94 (1982) 713; Angew.Chem, 1nternat. Ed1t.Eng1. 21 (1982) 707; Angew.Chem.Suppl. 1982, 1575-1604; s.a. die dort zitierte Literatur.

111/ L.K. Bell, W.A. Hermann, G.W. Kriechbaum, H. Pfisterer, M.L. Ziegler, J.organometal.Chem. 240 (1982) 381.

$112 /$ R.J. Klingler, W.M. Butler, M.D. Curt1s, J.Amer.Chem.Soc. 97 (1975) 3535 .

/13/ w.I. Bailey Jr., M.H. Chisholm, F.A. Cotton, L.A. Rankel, J.Amer.Chem.Soc. $100 \quad(1978) 5764$.

114/ A.F. Wells, Structural Inorganic Chemistry, 4. Auflage, S. 234 ff., S. 674 und S. 679, Clarendon Press, Oxford 1975.

15/ Aufgrund der entsprechenden Xovalenzradien sind fur $\mathrm{P}-\mathrm{C}$-Bindungen die folgenden Atomabstande zu erwarten: Einfachbindung $187 \mathrm{pm}$, Doppelbindung $167 \mathrm{pm}$, Dreifachbindung $153 \mathrm{pm} ;$ L. Pauling, Die Natur der Chemischen B1ndung, 3. Auflage, S. $214 \mathrm{ff.}$ Verlag Chemie, Weinheim 1976 ; vg1. D.E.C.Corbridge, The Structural Chenistry of Phosphorus, Elsevier Sclentific Publishing Company, Ansterdan 1974.

$116 / \mathrm{P}-\mathrm{C}-\mathrm{E}$ infachbindungsiăngen wurden experimentell zu 182$186 \mathrm{pm}$ gefunden $/ 14 /$. Wie Struktur des freien Liqanden 1st bekannt: H.Cbertanner, G.Becker, G.Gresser, J.Mbl.Spectrosc. 75 (1981) 283.

117/ Neuere zusammenfassung mit theoretischen Betrachtungen Uber die Stereochemle von Acetylen-Komplexen: D.M. Hoffman, R. Hof fmann, C.R. Fisel, J.Amer.Chem.Soc. 104 (1982) 3858 .

\section{$-525-$}

/18/ D.S. Ginley, C.R. Bock, M.S. Wrighton, B. Fischer, D.L. Tipton, R. Ban, J.Organometal.Chem. 157 (1978) 41.

/19/ W.G. S1y, J.Arer.Chem.Soc. 81 (1959) 18; D.A. Brown, J.Chem.Phys. 33 (1960) 1037.

120/ D. Seyferth, J.S. Merola, J.Amer.Chem.Soc. 100 (1978) 6783; C.Seyferth, J.S.Merola, R.S.Henderson, Organcinetallics 1 (1982) 859 .

/21/ P.J. Sullivan, A.L. Rheingold, Organometallics 1 (1982) 1547.

122/ F.A. Cotton, w. Schwotzer, Angew.Chem. 94 (1982) 652; Angew.Chem. 2nternat.Ed1t. Engl. 21 (1982) 629

123/ W.A. Herrmann, H. Biersack, Chem.Ber. 110 (1977) 896

/24/ W.A. Herrmann, H. Blersack, K.x. Mayer, B. Reiter, Chem. Ber. 113 (1980) 2655.

/25/ W.P. Fehlhamer, W.A. Herrmann, K. Ofele, "Metallorganische Komplexverbindungen" in: Handbuch der Präparat1ven Anorganischen Chemle (G. Brauer, Hrsgb.), 3. Auflage, 3. Band, S. 1799, Ferdinand Enke Verlag, Stuttgart 1981.

/26/ G.M. Sheldrick, SHELXTL Programm, Version 1980, Anorganisch-chemisches Institut, Universitalt Göttingen.

127/ International Tables for X-Ray Crystallography, Vol. IV, S. 99. Kynoch Press, B1rmingham (GroBbritannien) 1974.

128/ A.S.Foust, M.S.Foster, L.F.Dah1, J.Amer.Chem.Soc. 91 (1969) 5633; A.S.Foust, C.F.Campana, J.D.Sinclair, L.F. Dahl, Inorg.Chem. 18 (1979) 3047.

129/ W.Berdesinski, B.Nuber, Neues Jahrb.Mineral. Abhandl. 104 (1966) 113. 